Bull. Austral. Math. Soc.

$20 \mathrm{M} 10,20 \mathrm{M} 18$

VoL. 43 (1991) [463-466]

\title{
A NOTE ON SHORTLY CONNECTED INVERSE SEMIGROUPS
}

\section{S.M. Goberstein}

\begin{abstract}
Shortly connected and shortly linked inverse semigroups arise in the study of inverse semigroups determined by the lattices of inverse subsemigroups and by the partial automorphism semigroups. It has been shown that every shortly linked inverse semigroup is shortly connected, but the question of whether the converse is true has not been addressed. Here we construct two examples of combinatorial shortly connected inverse semigroups which are not shortly linked. One of them is completely semisimple while the other is not.
\end{abstract}

\section{INTRODUCTION}

Let $S$ be an inverse semigroup. The semilattice of idempotents of $S$ will be denoted by $E_{S}$ and the natural partial order on $S$ by $\leqslant$. If $x \in S$, we denote by $\langle x\rangle$ the monogenic inverse subsemigroup of $S$ generated by $x$. Suppose that $x \in S$ and $g \in E_{S}$ are such that $g<x x^{-1}$ and there is no $f \in\langle x\rangle$ satisfying $g<f<x x^{-1}$. Then we say that $g$ is $x$-covered by $x x^{-1}$. Now take $a \in S$ and $e<a a^{-1}$. Suppose that for some $n \geqslant 1$, there exist $e_{0}, e_{1}, \ldots, e_{n} \in E_{S}$ such that $e=e_{0}<e_{1}<\ldots<e_{n}=a a^{-1}$ and for any $i=1, \ldots, n, e_{i-1}$ is $a_{i}$-covered by $a_{i} a_{i}^{-1}$ where $a_{i}=e_{i} a$ (and hence $\left.a_{i} a_{i}^{-1}=e_{i}\right)$. Then $\left(e_{0}, e_{1}, \ldots, e_{n}\right)$ is called a short bypass from $e$ to $a a^{-1}$. If for any $a \in S$ and any $e<a a^{-1}$, there exists a short bypass from $e$ to $a a^{-1}, S$ is said to be shortly connected. Shortly connected inverse semigroups were introduced in [1] due to their remarkable role in the study of determinability of inverse semigroups by the lattices of inverse subsemigroups and by the partial automorphism semigroups (see [1]). However their description appeared to be rather technical. Thus we also introduced in [1] the class of shortly linked inverse semigroups which could be described much more conveniently and which are also determined (under natural additional assumptions) by their lattices of inverse subsemigroups.

Let $S$ be an inverse semigroup. For $a \in S$ and $e<a a^{-1}$, set $F_{e, a}=\{f \in\langle a\rangle: e<$ $\left.f \leqslant a a^{-1}\right\}$. We say that $S$ is shortly linked if for any $a \in S$ and $e<a a^{-1}, F_{e, a}$ is finite. It follows that any inverse semigroup $S$ such that $E_{\langle a\rangle}$ is finite for every $a \in S$, is shortly linked (for example, periodic inverse semigroups and, in particular, semilattices are shortly linked). It was shown in [1] that any shortly linked inverse semigroup

Received 19 June 1990

Copyright Clearance Centre, Inc. Serial-fee code: 0004-9729/91 $\$ \mathbf{A 2 . 0 0 + 0 . 0 0}$. 
is shortly connected. When the results of [1] were reported at several seminars, the question was raised whether the converse is also true. The purpose of this note is to show that, in general, the converse does not hold; that is, there exist shortly connected inverse semigroups which are not shortly linked.

\section{The EXAMPLES}

ExAmple 1: Let $E=\left\{e_{0}, e_{1}, e_{2}, \ldots, f_{0}, f_{1}, f_{2}, \ldots, 0\right\}$ be a semilattice whose diagram is shown in Figure 1. Let $S=T_{E}$ be the Munn semigroup of the semilattice $E$ [2, Section V.4]. As usual, we identify $E_{S}$ with $E$. It is easy to see that for all integers $m, n \geqslant 0, E e_{m} \cong E e_{n} \not E f_{m} \cong E f_{n}$.

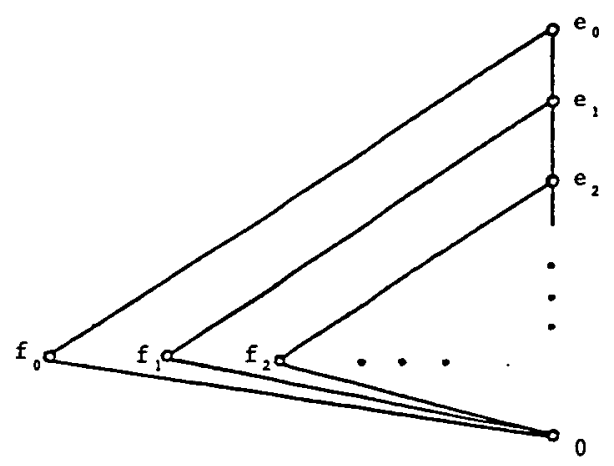

Figure 1

Moreover, the only isomorphism of $E e_{m}$ onto $E e_{n}$ is $\phi_{m, n}$, defined as follows (see [2, Example V.4.6]):

$$
0 \phi_{m, n}=0, \quad f_{k} \phi_{m, n}=f_{k-m+n}, \quad e_{k} \phi_{m, n}=e_{k-m+n} \quad(k \geqslant m)
$$

and the restriction of $\phi_{m, n}$ to $E f_{m}$ is the only isomorphism of $E f_{m}$ onto $E f_{n}$. It is easily seen that $S$ is a combinatorial (that is, $\mathcal{H}$-trivial) inverse semigroup with exactly three D-classes: $D_{0}, D_{f_{0}}$ and $D_{e_{0}}$ (see [2, the proof of Proposition V.6.1]). More specifically, $D_{0}=\{0\}, D_{0} \cup D_{f_{0}}$ is the combinatorial Brandt semigroup [3, Section II.3] whose set of nonzero idempotents is $\left\{f_{0}, f_{1}, f_{2}, \ldots\right\}$, and $D_{e_{0}}$ is the bicyclic semigroup generated by $a=\phi_{0,1}$ (so that $a a^{-1}=e_{0}$ and $a^{-1} a=e_{1}$ ). Since the set $F_{0, a}$ is infinite, $S$ is not shortly linked. On the other hand, for any $m \geqslant n \geqslant 0$ and any $x \in D_{e_{0}}$ and $y \in D_{f_{0}}$ such that $x x^{-1}=e_{m}$ and $y y^{-1}=f_{m},\left(0, f_{m}, e_{m}\right)$ is a short bypass from 0 to $x x^{-1},\left(f_{n}, e_{n}, e_{n+1}, \ldots, e_{m}\right)$ is a short bypass from $f_{n}$ to $x x^{-1}$, and $\left(0, f_{m}\right)$ is a short bypass from 0 to $y y^{-1}$. Therefore $S$ is shortly connected. 
Since the semigroup $S$ in Example 1 contains a bicyclic subsemigroup, it is not completely semisimple. In the next example we construct a completely semisimple shortly connected inverse semigroup which is not shortly linked.

EXAmple 2: Let $E$ be a semilattice given by the diagram in Figure 2.

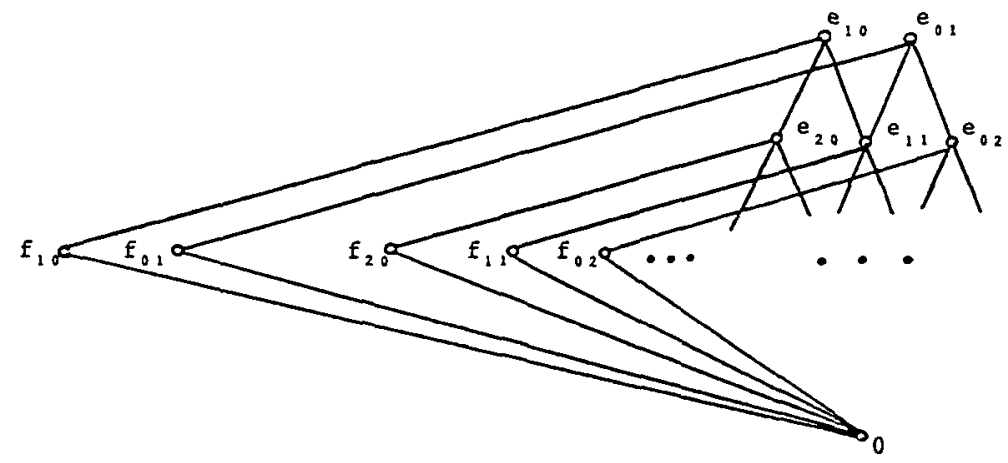

Figure 2

Thus $\left\{e_{10}, e_{01} ; e_{20}, e_{11}, e_{02} ; \ldots\right\}$ is the semilattice of idempotents of the free monogenic inverse semigroup where for each $m \geqslant 1,\left\{e_{m-k, k}: k=0,1, \ldots, m\right\}$ is the set of all idempotents of that semigroup of weight $m$ [3, Sections IX.1 and IX.2] (we denote by $e_{m n}$ the idempotent which can be uniquely written in the form $e_{m} f_{n}$ in the notation of $[3, \mathrm{p} .408])$. Moreover, $\left\{f_{10}, f_{01}, f_{20}, f_{11}, f_{02}, \ldots, 0\right\}$ is a primitive semilattice [3, Definition II.4.2] and each $f_{m n}$ is covered in $E$ by $e_{m n}(m, n \geqslant 0 ; m+n>0)$. Define a mapping $\alpha: E e_{10} \rightarrow E e_{01}$ as follows:

$$
0 \alpha=0, \quad f_{m n} \alpha=f_{m-1, n+1}, \quad e_{m n} \alpha=e_{m-1, n+1} \quad(m \geqslant 1, n \geqslant 0) .
$$

It is easily seen that $\alpha$ is an isomorphism of $E e_{10}$ onto $E e_{01}$. Let $S$ be the full inverse subsemigroup of $T_{E}$ generated by $\alpha$. Since $F_{0, \alpha}$ is infinite, $S$ is not shortly linked. On the other hand, one can easily check that $S$ is a shortly connected combinatorial inverse semigroup (we omit the details). Since $S$ does not contain a bicyclic subsemigroup, it is completely semisimple.

\section{REFERENCES}

[1] S.M. Goberstein, 'Inverse semigroups with isomorphic partial automorphism semigroups', J. Austral. Math. Soc. (Ser. A) 47 (1989), 399-417.

[2] J.M. Howie, An introduction to semigroup theory (Academic Press, London, 1976).

[3] M. Petrich, Inverse semigroups (Wiley, New York, 1984). 
Department of Mathematics and Statistics

California State University, Chico

Chico, California 95929-0525

United States of America 